\title{
FOTOBIORREACTOR: HERRAMIENTA PARA CULTIVO DE CIANOBACTERIAS
}

\section{PHOTOBIOREACTOR: TOOL FOR MASS CULTIVATION OF CYANOBACTERIA}

\author{
Luis Guillermo Ramírez Mérida ${ }^{1}$, Leila Queiroz Zepka², Eduardo Jacob-Lopes ${ }^{2}$ \\ ${ }^{1}$ Unidad de Biotecnología Aplicada, Departamento de Biología, Facultad de Ciencias y Tecnología (FACYT), \\ Universidad de Carabobo (UC), Av. Universidad, Valencia 2002, Edo.Carabobo, Venezuela. \\ ${ }^{2}$ Departamento de Ciencia y Tecnología de Alimentos, Universidad Federal de Santa María (UFSM), \\ Av. Roraima 1000, 97105-900, Santa María-RS, Brasil.
}

Emails: luisguillermolgrm@hotmail.com; zepkaleila@yahoo.com.br; jacoblopes@pq.cnpq.br

ReSUmen

$\mathrm{L}$ as cianobactérias son organismos eficientes en la conversión de energía solar y producen una gran variedad de metabolitos. En la actualidad son el centro de atención para la producción de biocombustible, son usadas como biofertilizantes, control de contaminación ambiental y como fuente de nutrientes en alimentación humana y animal. Con el fin de proporcionar crecimiento y aprovechar el potencial de las cianobacterias, se requieren fotobiorreactores eficientes. Aunque se han propuesto muchos tipos de fotobiorreactores, no existe un reactor ideal, solo unos pocos pueden utilizarse para la producción de biomasa de cianobacterias. De hecho, la elección del fotobiorreactor más adecuado depende de la situación, ya que tanto las especies de algas disponibles y el destino final jugarán un papel importante. Uno de los principales factores que limita su aplicación práctica en cultivos de biomasa es la transferencia de masa. Por esto, entender el coeficiente de transferencia de masa en los fotobiorreactores es necesario para una operación eficiente del cultivo de biomasa en cianobacterias. En esta revisión, se discuten varios tipos de fotobiorreactores muy promisorios para la producción de biomasa de cianobacterias.

Palabras clave: Biomasa, transferencia de masa, fotobiorreactor, cianobacteria, microalga.
Abstract

Cyanobacteria are efficient organisms in the conversion of solar energy and also produce a wide variety of metabolites. At the present time they are intensively used as biofertilizers, for the biofuel production, environmental pollution control as well as a nutrients source in human and animal food. With the aim to provide growth and use the cyanobacteria's potential, efficient photobioreactors are required. Although many photobioreactor types have been proposed, just a few can be used for the biomass production of cyanobacteria. One of the main factors that limits the practical application of photobioreactors in biomass cultivation is mass transfer. Therefore understanding the mass transfer coefficient is of a great importance for efficient operation of the biomass cultivation in cyanobacteria. In this review, some very promising types of photobioreactors for cyanobacterial biomass production are discussed.

Key words: Biomass, mass transfer, photobioreactors, cyanobacteria, microalgae.

\section{INTRODUCCIÓN}

L as cianobacterias conocidas como alga o bacteria verde-azul, son organismos que pueden obtener su energía mediante fotosíntesis oxigénica. Son ubicuas y crecen en una amplia variedad de condiciones acuáticas y terrestres. Son importantes en el ciclo de nitrógeno

Recibido: 02-junio-2013. Recibido en forma corregida: 25-julio-2013. Aceptado: 28-septiembre-2013.

Publicado como ARTÍCULO DE REVISIÓN en Ciencia y Tecnología 6(2): 9-19

Julio-Diciembre de 2013

ISSN 1390-4051 impreso; ISSN 1390-4043 electrónico

(C) Ciencia y Tecnología. UTEQ. Quevedo-Ecuador marino, siendo los únicos procariotas que en condiciones aeróbicas pueden fijar dióxido de carbono $\left(\mathrm{CO}_{2}\right)$ igual que las algas eucariotas y plantas (Kumar et al., 2011). Las cianobacterias proveen moléculas bioactivas, su potencial biotecnológico ha sido estudiado por su capacidad de sintetizar ácidos grasos poliinsaturados tipo EPA (Eicosapentanoico) y DHA (Docohexanoico), útiles en formulación de alimentos como leches infantiles, presentando un elevado potencial para la aplicación en diversas áreas de la nutrición humana, 
alimentación animal, así como efecto antiinflamatorio, antioxidante y terapéutico (Bhagavathy y Sumathi 2012; Castine et al., 2013). Estos productos se emplean para aliviar o evitar algunas sintomatologías, como las provocadas por el estrés cotidiano, el sobrepeso o la inflamación, entre otras.

En general, son antioxidantes que reducen la intensidad del estrés oxidativo; sin embargo, existe un gran número de cianobacterias que hasta la fecha no se han explorado ni explotado su posible potencial, por lo que representan una oportunidad única para obtener tanto metabolitos conocidos a bajo costo, como nuevos metabolitos. Cianobacterias del género Spirulina son ampliamente utilizados como protectores antioxidantes en diversos modelos toxicológicos, debido a su alto contenido de compuestos como $\beta$-caroteno, vitaminas, ácidos grasos poliinsaturados $\omega-3$ y $\omega-6$ y ficobiliproteínas que son pigmentos fotosintéticos siendo la ficocianina el principal de ellos (Sharma et al., 2007).

La ficocianina de Arthrospira puede ser utilizada en ensayos de inmunofluorescencia (Perez-Garcia et al., 2011). Su uso es altamente beneficioso e inocuo, se ha demostrado que Spirulina maxima y Pseudanabaena tenuis poseen efectos antioxidantes y antiinflamatorios en diferentes modelos que generan estrés oxidativo y daño celular (Gallardo-Casas et al., 2011). Debido a su estabilidad, la ficocianina también se utiliza en formulaciones cosméticas y colorantes en alimentos.

La abundante fuente de antioxidantes que poseen las cianobacterias, las hace candidatas para uso en procesos biológicos, especialmente en el envejecimiento, así como en los cambios degenerativos de diferentes órganos (Datla, 2011). Spirulina posee alto contenido de proteínas, ácidos grasos esenciales, con fracción grasa que puede alcanzar $70 \%$ en peso seco, carbohidratos de alta digestibilidad, y vitaminas (A, B, C, E).

Géneros como Spirulina y Arthrospira, debido a su riqueza en proteínas y su perfil de aminoácidos se puede utilizar como nutracéutico o ser incluido en alimentos funcionales para prevenir algunas enfermedades y daños en las células y/o tejidos. Los efectos beneficiosos de los nutracéuticos y de los alimentos funcionales se han atribuido a polifenoles, ácidos grasos poliinsaturados (PUFAs), terpenos, clorofila y pigmentos accesorios del aparato fotosintético de las cianobacterias (Conroy et al., 2011; Cano et al., 2012).

Además la generación de algunas proteínas, pequeños péptidos y aminoácidos en contribuyen a proporcionar una alta cantidad de fósforo; combatir la hipertensión y la reducción de niveles de colesterol, ayudar en la formación y regeneración de células sanguíneas juntamente con el hierro (por su contribución en vitaminas del complejo B por lo que puede usarse en el tratamiento preventivo de anemias) y estimular la liberación de sustancias que controlan la saciedad, resultando en la reducción del apetito (Becker, 2004; Raposo et al., 2013). Ya existen en los anaqueles algunas pastillas con marca comercial realizadas a base de Spirulina o Arthrospira, como es el caso de los productos comerciales que se pueden evidenciar en los siguientes enlaces: http://spirulina.greennutritionals. com.au/; http://www.natue.com.br/clorela-e-spirulina/

Por otra parte, se ha observado que polisacaridos aislados de Spirulina sp., se unen a la alquil hidroperóxido reductasa $(\mathrm{AhpC})$ y ureasa de Helicobacter pylori reduciendo en $99 \%$ la actividad de unión a la mucina de la pared celular gástrica de ratones BALN/c; confirmando su posible efecto protector contra la colonización de $H$. pylori (Loke et al., 2007).

Además despiertan la atención por su aplicación ambiental, tanto en producción de biocombustible como en biorremediación; debido a que las cianobacterias, podrían ofrecer más biocombustible que los cultivos de semillas oleaginosas utilizando menos agua y tierra, además de emplear energía solar, sin embargo, las condiciones de cultivo de cianobacterias deberían ser optimizadas para maximizar la síntesis de lípidos (Chisti, 2007).

En biorremediación, las cianobacterias producen exopolisacaridos y sustancias floculantes, permitiéndoles proliferar en ecosistemas de aguas residuales y dominar medios eutrofizados aunado a su capacidad de reducir $\mathrm{CO}_{2}$ y otros compuestos contaminantes (Gupta et al., 2013).

La obtención de estos y otros productos requieren condiciones, equipamiento (fotobiorreactor), con un microambiente adecuado para que el cultivo pueda generar cantidades importantes de biomasa. Diseñar, escoger y utilizar un fotobiorreactor adecuado para cianobacterias, es un punto crucial para su cultivo ya que poder lograr una actividad productiva controlada permitirá que los rendimientos de biomasa, metabolitos primarios y/o secundarios puedan ser altos y con relativa alta especificidad para su obtención a mediana y gran escala. Se debe considerar en el diseño y selección del fotobiorreactor un efectivo y eficiente suministro de luz y $\mathrm{CO}_{2}$, remoción del oxígeno generado fotosintéticamente ya que este puede inhibir el metabolismo celular o dañar el cultivo, una tecnología razonable para ampliación (escalamiento), control de temperatura, presión, mezclado y $\mathrm{pH}$.

Esta revisión tiene como objetivo suministrar las características más destacadas, limitaciones, propiedades, acontecimientos y formas de acción de los diferentes fotobiorreactores necesarios para el cultivo de cianobacterias. 


\section{REVISIÓN DE LITERATURA}

Parámetros de procesamiento a tomar en cuenta en el diseño de fotobiorreactores

A pesar del desarrollo de diferentes fotobiorreactores, pocos utilizan efectivamente la luz solar como energía para el cultivo microbiano. Un problema frecuente en diseño de fotobiorreactores es la provisión óptima de energía solar al aire libre, que todas las células tengan la misma exposición de luz, suministrar una relación área superficial/volumen (S/V) grande, que ocupe menos espacio terrestre, rápida transferencia de masa $\mathrm{y}$ que logre una mayor productividad (Janssen et al., 2000).

\section{Suministro de luz}

En sistemas abiertos o cerrados de cultivo de cianobacterias, la fuente de luz y la intensidad de energía son factores que afectan su desarrollo y crecimiento. En cultivos de sistemas abiertos, la luz solar es la principal fuente de energía; mientras que para sistemas de cultivo cerrados hay diferentes fuentes luminosas a utilizar como: Lámparas de tungsteno o halógeno, diodos emisores de luz (LED), lámparas fluorescentes, fibra óptica y láser. Estos sistemas de energía encarecen el diseño del fotobiorreactor (Janssen et al., 2000).

La intensidad de luz puede afectarse por factores como: Distancia entre la energía lumínica y el fotobiorreactor, geometría del fotobiorreactor, longitud de onda, aumento de concentración celular, formación de biopelículas en las paredes y formación de productos, provocando debido a efecto de sombreado la formación de dos zonas (oscura e iluminada). En la zona iluminada, las células son expuestas a la luz necesaria para producir fotosíntesis; en la zona oscura las células reciben poca o casi nada de luz para su metabolismo (Janssen et al., 2000). Una forma de reducir el efecto planteado arriba sería acortar la distancia de incidencia de luz, sin embargo, esto no se lograría con luces convencionales ya que el contacto cercano con las paredes del fotobiorreactor generará calor el cual dañaría el cultivo.

Por esto, las investigaciones en la actualidad se centran en el uso de energía lumínica que no genere calor, pero estos sistemas tienden a elevar los costos. Melnicki et al. (2013) diseñaron un fotobiorreactor con ajustes de luz tipo LED automática utilizando un control computarizado de retroalimentación, mejorando los sistemas de cultivos de cianobacterias, alcanzando nuevas capacidades para la investigación fisiológica y biología de sistemas. Tamburic et al. (2011) diseñaron un fotobiorreactor de superficie plana (flat plate) con iluminación blanca fría, proporcionada por un panel de diodos emisores de luz que presentó alta eficiencia fotoquímica. Por su parte, Sepúlveda (2011) propuso tres sistemas de iluminación para fotobiorreactores de columna de burbujeo: i) Lámpara fluorescente que irradiaba sobre el fotobiorreactor desde un solo punto, ii) Tira comercial flexible de LEDS colocada en forma de espiral rodeando la parte externa del fotobiorreactor y iii) Tira comercial flexible de LEDS colocada en forma de espiral rodeando la pared interna de un tubo hueco transparente colocado en el interior del fotorreactor. La biomasa generada con la configuración de iluminación de la tira de LEDS externa fue de 112 millones de células $\mathrm{mL}^{-1}$, aproximadamente el doble de la biomasa generada con los otros dos sistemas utilizados esto debido a la mayor trayectoria de fotones dentro del fotobiorreactor. Sin embargo, las investigaciones se encaminan a lograr un sistema efectivo, económico y sustentable en gasto energético.

La radiación lumínica en que las cianobacterias son capaces de realizar fotosíntesis y crecimiento celular está entre 400 y $700 \mathrm{~nm}$, con una iluminancia óptima de 11 klux (Jacob-Lopes et al., 2008). Si la cantidad de luz irradiada es insuficiente, disminuirá considerablemente la velocidad de crecimiento del microorganismo; pero si hay exposición excesiva con alta intensidad por largos periodos de luz en el medio se producirá fotoinhibición, fenómeno donde los fotosistemas del microorganismo se dañan e inactivan procesos de transporte celular.

Para evitar daño en los sistemas fotosintéticos por exceso de luz irradiada, se diseñan sistemas que distribuyan luz en mayor superficie (Torzillo et al., 2003). Esto hace que la geometría del reactor reduzca la atenuación de luz en el cultivo. Fernández et al. (2001) estudiaron el efecto de geometría plana y circular en relación a la penetración de luz en cultivos con concentraciones similares de microalgas, evidenciando que la geometría circular permite mejor penetración de luz que la geometría plana proporcionando mayor fracción de volumen del reactor para recibir iluminación, sin embargo, la geometría plana ayuda en una distribución uniforme de luz.

Con herramientas de biología molecular, se puede disminuir el tamaño de las antenas fotosintéticas celulares reduciendo la absorción de luz dando mayores rendimientos fotosintéticos (Kwon et al., 2013). El fotoperiodo al que se somete el cultivo también es importante en el diseño del proceso ya que la fotosíntesis conlleva reacciones de luz y oscuridad. La duración de ciclos luz/oscuridad son determinantes en el desarrollo de fotobiorreactores para ser considerado en la producción de biomasa y en la absorción del $\mathrm{CO}_{2}$. Jacob-Lopes et al. (2009) mostraron una reducción lineal evidente en la producción de biomasa y en la fijación de $\mathrm{CO}_{2}$ en microalgas con disminución de la duración del periodo 
de luz, exceptuando el ciclo 12:12 (noche:día). Ellos obtuvieron reducciones de $99.69 \%$ en tasas de fijación de carbono en comparación con cultivos bajo iluminación continua.

\section{Provisión y transferencia de masa de $\mathrm{CO}_{2}$}

La fuente de carbono usual para el cultivo fotosintético de cianobacterias es el gas $\mathrm{CO}_{2} \mathrm{o}$ su forma disuelta bicarbonato $\left(\mathrm{HCO}_{3}\right)$ difundido en el medio de cultivo. Aun cuando el medio de cultivo esté bien mezclado, la simple difusión del $\mathrm{CO}_{2}$ del aire en el agua no es suficiente para reemplazar el consumido por los microorganismos. El $\mathrm{CO}_{2}$ es generalmente introducido al fotobiorreactor por inyección continua o intermitente en la parte inferior del recipiente, el suministro de $\mathrm{CO}_{2}$ en algunas cianobacterias produce un incremento de biomasa, sin embargo, a su vez depende de muchos parámetros que incluyen la lenta velocidad de difusión del $\mathrm{CO}_{2}$ en el medio líquido, $\mathrm{pH}$, turbulencia, temperaturas y densidades celulares (Jaiswal y Kashyap, 2002).

Se han descrito cepas de cianobacterias con elevados requerimientos de $\mathrm{CO}_{2}$ equivalentes al 38\% en Spirulina sp. (de Morais y Costa, 2007). Estas cepas han sido comparadas con variedades silvestres capaces de crecer a un bajo nivel de $\mathrm{CO}_{2}$ de $0.03 \%$ presente en el aire (Reinhold et al., 1991). Muchos diseños de fotobiorreactores en campo abierto, están adoptando el suministro externo de $\mathrm{CO}_{2}$ para mejorar el cultivo ya que algunos autores reflejan que esos niveles $(0.03 \%)$ de $\mathrm{CO}_{2}$ no son suficientes para generar alta productividad de biomasa (Posten y Schaub, 2009).

Los límites máximos y mínimos de $\mathrm{CO}_{2}$ necesarios no están bien definidos pero en la práctica se usa comúnmente aireación con $5-15 \%$ de $\mathrm{CO}_{2}$, aunque esto varía en relación a la cepa microbiana (Jaiswal y Kashyap, 2002). El suministro continuo de $\mathrm{CO}_{2}$ es la manera idónea de mantener el cultivo, sin embargo el $\mathrm{CO}_{2}$ comercial es costoso, una manera de contrarrestar esto es usar $\mathrm{CO}_{2}$ de desecho industrial, o el empleo de biogás a partir del uso de microorganismos que generen digestión anaerobia de residuos agroindustriales, el uso de $\mathrm{CO}_{2}$ generado en la fermentación del vino (Proyecto desarrollado por la empresa Aeon Biogroup, Catapilco, Chile) o el diseño de fotobiorreactores con sistemas de aspersión y mezclado que permita que las burbujas de gas se retengan el tiempo suficiente para ser absorbido en el medio líquido, pero el gas en burbujas muy finas, debido a su largo tiempo de residencia en el fluido, alcanzará el equilibrio con el líquido y por lo tanto contribuirá poco a la transferencia de masa (Markou y Georgakakis, 2011).

Entre los sistemas más comúnmente utilizados se encuentran la alimentación directa del gas en columnas de burbujeo o reactores airlift (Chen et al., 2011). La transferencia de masa gas-líquido en fotobiorreactores cerrados ha sido direccionada siguiendo desarrollos similares para cultivo abierto tipo raceway (Molina et al., 1999). El coeficiente volumétrico de transferencia de masa $\left(\mathrm{K}_{\mathrm{L}} \mathrm{a}\right)$ es una de las características que determina la capacidad del reactor para sostener el crecimiento celular óptimo. El comportamiento de $\mathrm{K}_{\mathrm{L}}$ a y la velocidad de crecimiento celular varían en diferentes zonas del flujo de líquido. Estas zonas de flujo de líquido en fotobiorreactores pueden dividirse en zona de flujo de burbujas, y zona de transición heterogénea y dependerá de la velocidad del gas. En la zona de flujo de burbuja, la capacidad de retención del gas, el área interfacial y la $\mathrm{K}_{\mathrm{L}}$ a son proporcionales a la velocidad superficial del gas. Aunque la disminución en el área interfacial comienza con el movimiento que va desde la zona de transición a la zona heterogénea, sin embargo, la capacidad de retención del gas y $\mathrm{K}_{\mathrm{L}} \mathrm{a}$ alcanzan la estabilidad. Ciertos tipos de mezclado, con relación a la tensión de cizallamiento, puede ser la razón de la caída en la tasa de crecimiento específica. Además, el $\mathrm{K}_{\mathrm{L}}$ a aumenta dentro de un rango de tamaño de burbuja en columnas de burbujeo y reactores airlift. Burbujas pequeñas $(<2 \mathrm{~mm}$ diámetro) causan más daño celular que burbujas mayores ( $\sim 10 \mathrm{~mm}$ diámetro) (Chisti y Jauregui-Haza, 2002). Por tanto, la mezcla, el tamaño de la burbuja, la velocidad superficial del gas y la presión de $\mathrm{CO}_{2}$ deben ajustarse a los requerimientos de cada tipo específico.

Por otra parte, en condiciones de alta temperatura, las pérdidas por fotorrespiración aumentan sustancialmente. Esto ocurre ya que, a altas temperaturas ( $40^{\circ} \mathrm{C}$ en adelante), la solubilidad del $\mathrm{CO}_{2}$ disminuye drásticamente, resultando en una menor disponibilidad del $\mathrm{CO}_{2}$ respecto del $\mathrm{O}_{2}$ en el sitio activo de la enzima. Así, condiciones de cultivo con rango de temperatura entre $30-35^{\circ} \mathrm{C}$ y $15-20 \%$ de $\mathrm{CO}_{2}$, favorece el crecimiento celular de Aphanothece microscopica Nägeli asociado con la fijación de carbono (Jacob-Lopes et al., 2008).

\section{Acumulación de oxígeno}

El oxígeno es un producto de la fotosíntesis, cuando queda atrapado en el medio de cultivo causa un efecto tóxico reduciendo la eficiencia fotosintética, generando inhibición del crecimiento incluso a concentraciones altas de $\mathrm{CO}_{2}$. Por esto, se necesita un sistema eficaz de desgasificación para remover el oxígeno formado. La acumulación de oxígeno es un serio problema en fotobiorreactores que tienen un pobre intercambio de gas como los sistemas tubulares dispuesto de forma horizontal.

El problema de acumulación de oxígeno aumenta cuando un fotobiorreactor tubular helicoidal sufre 
escalamiento en el sistema de iluminación. Por lo tanto, es necesario tener una unidad separada de desgasificación, en muchas oportunidades esta unidad puede estar acoplado con el sistema de inyección airlift (Soletto et al., 2008), ya que la inyección de gas desde el fondo del fotobiorreactor favorece el mezclado, suministra suficiente $\mathrm{CO}_{2}$ y dependiendo de la altura del fotobiorreactor, se logra una eficiente remoción de oxígeno disuelto en el medio. La remoción de exceso de oxígeno es un problema de transferencia de masa parecido al de suministro de $\mathrm{CO}_{2}$, las principales formas de controlar este fenómeno son: Disminuir la presión de oxígeno, mayor agitación y altas temperaturas. Una solución al problema es aumentar la turbulencia del medio. Por esto, fotobiorreactores de tanque agitado y vertical, serían más convenientes. Con el aumento en la velocidad del líquido, que depende de la entrada del gas, la concentración de oxígeno disuelto disminuye, mejorando la producción de biomasa aunque, mucha velocidad podría ocasionar daño y muerte celular (Barbosa et al., 2003).
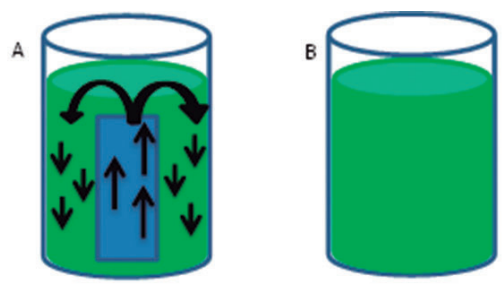

D

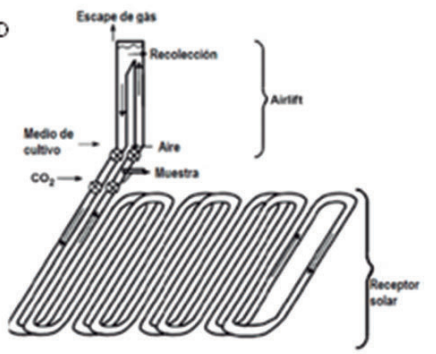

E
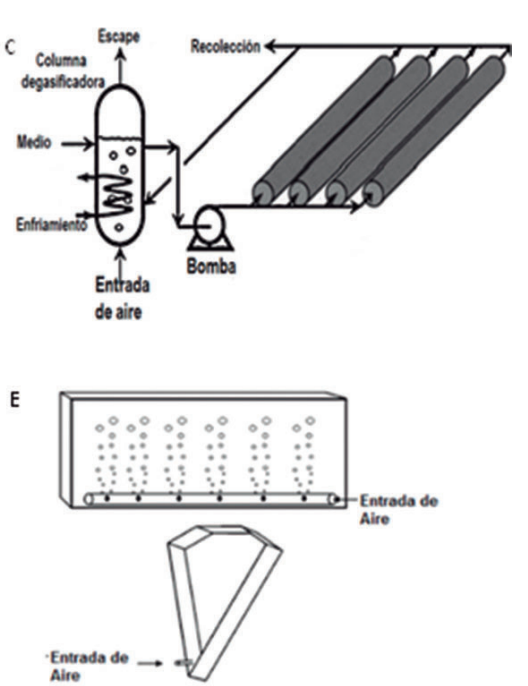

\section{Mezcla} (Ugwu et al., 2002).

Aire
Una vez que en el fotobiorreactor se alcancen las proporciones adecuadas del medio de cultivo y los parámetros fisicoquímicos requeridos, el sistema de mezclado llega a convertirse en un punto fundamental. El mezclado debe permitir condiciones homogéneas, favorecer la transferencia de nutrientes, dispersión uniforme de cianobacterias entre las zonas oscuras e iluminadas, transferencia de energía, romper gradientes de difusión en la superficie celular, retirar el oxígeno fotosintético generado, evitar estratificación térmica ya que puede existir amplitud térmica entre fondo y superficie del fotobiorreactor de hasta $8^{\circ} \mathrm{C}$ (Carvalho et al., 2006). Un buen mezclado puede aumentar la productividad hasta $40 \%$ en un fotobiorreactor tubular

Técnicas actuales de mezcla son generalmente insuficientes para lograr los requerimientos mencionados, ya que la turbulencia generada es al azar es decir, no todas las células se mezclan uniformemente entrando y saliendo de las zonas oscuras e iluminadas.

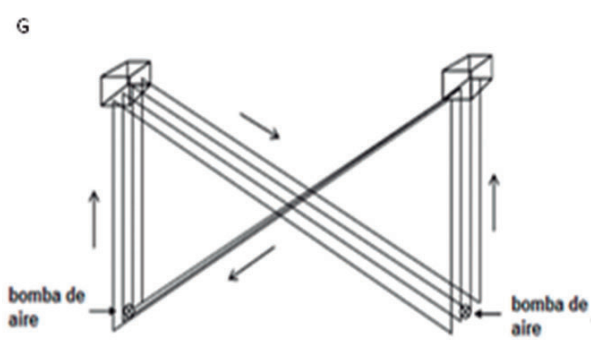

G

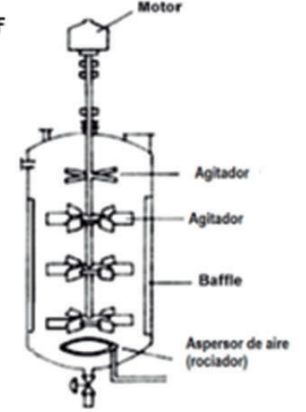

aire

aire

Figura 1. Esquema representativo de diferentes configuraciones de fotobiorreactores. Sistema airlift (A), columna de burbujeo (B), tubular horizontal (C), tubular helicoidal (D), superficie plana (flat plate) (E), tanque agitado (F), tipo hibrido (G). Modificado de Carvalho et al. (2006) 
Mezclas inadecuadas promueven formación de aglomerados celulares que disminuyen la velocidad de transferencia de masa comprometiendo la viabilidad celular. Por esto, el continuo movimiento celular garantiza altas producciones de biomasa (Sato et al., 2010). Entre los sistemas de mezcla tenemos la bomba, agitación mecánica y mezcla de gases que trabajan solos o en combinación. El sistema de bomba, forma una corriente de recirculación que tiende a dañar la célula. El sistema de mezcla de gases como el empleado en columna de burbujeo, causa menos daños que los del sistema anterior. En éste se observa el sistema airlift, aquí la mezcla se alcanza por flujo de fluidos por burbujeo de aire dentro de un tubo central de subida, que luego se distribuye bajando por la parte exterior del tubo central, creando una circulación natural (Figura 1A) (Chisti y Jauregui-Haza, 2002).

Aunque este sistema parece causar menos daño celular, no está libre de estrés por cizallamiento, daños celulares por efecto hidrodinámico se reportaron en fotobiorreactores de columnas de burbujeo y airlift (Pruvost et al., 2006; Reyna-Velarde et al., 2010). La agitación mecánica donde un tubo central con dos o más hojillas con acción al fondo del reactor, es frecuente en reactores de un solo vaso. Buscando métodos menos dañinos a las células, existen sistemas híbridos donde se hace pasar una corriente de aire enriquecida con $\mathrm{CO}_{2}$ a través de un tubo central dentro de las cuchillas o aspas del agitador mecánico donde el flujo entra por medio de pequeños capilares (Singh y Sharma, 2012).

\section{Previsiones generales para escalamiento}

Molina et al. (1999) sugieren que al escalar un fotobiorreactor tubular, el diámetro de la columna no sea mayor a $0.1 \mathrm{~m}$ y longitud continua de $80 \mathrm{~m}$ con flujo de velocidad de $0.3-0.5 \mathrm{~m} \mathrm{~s}^{-1}$. Por su parte Sánchez et al. (2000) indican para un fotobiorreactor tubular vertical un diámetro de tubo no mayor a $0.2 \mathrm{~m}$ para que la disponibilidad de luz no se vea reducida. Sin embargo, estos diámetros pueden aumentarse si el reactor es diseñado con un sistema de iluminación interna. Las dificultades en el escalamiento surgen ya que los volúmenes de las zonas iluminadas y oscuras cambian a medida que aumenta el diámetro del tubo.

Es adecuado el uso de bases y tapas en acero inoxidable ya que además de resistir temperaturas de esterilización, se puede fijar un intercambiador de calor mediante una chaqueta térmica Sánchez et al. (2000). La tapa superior debe permitir la entrada de dispositivos que midan $\mathrm{pH}$ y oxígeno disuelto, dar salida de gases y en la base estará la entrada de gases y salida de líquido. Estos dispositivos de entrada y salida de gases, deben acompañarse de filtros de membrana para garantizar la esterilidad.
Al aumentar la proporción superficie iluminada y volumen en el fotobiorreactor $(\mathrm{S} / \mathrm{V})$, mayor es la concentración celular en el cultivo; para incrementar la productividad volumétrica sin aumentar la intensidad de luz en superficie, se acorta el camino de luz disminuyendo el diámetro de la columna, si bien esto provoca inconvenientes ya que la fuerza motriz necesaria para mantener la turbulencia es inversamente proporcional al diámetro del tubo empleado (Chini et al., 1999).

\section{Otros factores}

Controlar el pH, en ciertos límites, se dificulta ya que éste se incrementa continuamente debido al consumo de carbono en la fotosíntesis. El pH afecta la polaridad de los compuestos del medio de cultivo así como la disponibilidad de nutrientes, $\mathrm{CO}_{2}$, hierro y ácidos orgánicos; puede ocurrir precipitación química de sales que contienen $\mathrm{CO}_{3}^{-2}$, OH- y $\mathrm{PO}_{4}^{-3}$ llevando al deterioro del medio y posible daño celular. Existe un consenso general acerca de la preferencia de cianobacterias para el $\mathrm{CO}_{2}$ (como fuente de carbono inorgánico), ya que se controla fácilmente y produce cambios menores de $\mathrm{pH}$ (Jacob-Lopes et al., 2008).

\section{Tipos de fotobiorreactores}

Para que el cultivo de cianobacterias sea eficiente es necesario diseñar fotobiorreactores que transmitan la mayor cantidad posible de luz, esto implica alta relación superficie/volumen $(\mathrm{S} / \mathrm{V})$ del reactor. De esta forma, la eficiencia de un fotobiorreactor es determinada en base a la captación, transporte, distribución y uso de la luz (Zijffers et al., 2008). Se han diseñado diferentes fotobiorreactores con propiedades especificas (Cuadro 1) que pueden agruparse en tres tipos básicos: tubulares, de superficie plana (flat plate) y de tanque agitado. Los tubulares y de superficie plana son los más empleados considerando que utilizan luz solar.

\section{Fotobiorreactores tubulares}

Son los más fáciles de escalar incrementando la longitud, el número de tubos y uniendo múltiples unidades por medio de colectores, existen diferentes configuraciones:

A. Tubulares verticales. Formado por tubos verticales transparentes de vidrio o polietileno, para aprovechar la penetración de luz natural (Figura 1B). El rociador se ubica al fondo del vaso esparciendo pequeñas burbujas para una buena mezcla, suministro suficiente de $\mathrm{CO}_{2}$ y remoción de oxígeno. El airlift y la columna de burbujeo son ejemplo de ellos. González-Fernández et al. (2010) evaluaron el comportamiento de dos fotobiorreactores uno de tanque abierto y otro tubular cerrado, empleados 
Cuadro 1. Comparación de propiedades de diferentes fotobiorreactores utilizados en el cultivo de cianobacterias

\begin{tabular}{|c|c|c|c|c|c|c|c|c|}
\hline $\begin{array}{c}\text { Tipo } \\
\text { de } \\
\text { fotorreactor }\end{array}$ & $\begin{array}{c}\text { Eficiencia } \\
\text { de } \\
\text { mezcla }\end{array}$ & $\begin{array}{c}\text { Uso } \\
\text { eficiente } \\
\text { de luz }\end{array}$ & $\begin{array}{l}\text { Transfe- } \\
\text { rencia de } \\
\text { gas }\end{array}$ & $\begin{array}{l}\text { Estrés de } \\
\text { cizalla- } \\
\text { miento }\end{array}$ & $\begin{array}{l}\text { Escala- } \\
\text { miento }\end{array}$ & Perspectivas & Limitaciones & Referencia \\
\hline $\begin{array}{l}\text { Tanque } \\
\text { agitado }\end{array}$ & A & B-M & B-A & A & D & \multirow{3}{*}{$\begin{array}{l}\text { Presentan bajo } \\
\text { consumo de } \\
\text { energía, fácil de } \\
\text { esterilizar, reduce } \\
\text { fotoinhibición y } \\
\text { fotooxidación. }\end{array}$} & \multirow{3}{*}{$\begin{array}{l}\text { Requiere mate- } \\
\text { riales sofisti- } \\
\text { cados para su } \\
\text { construcción, } \\
\text { área pequeña } \\
\text { de superficie de } \\
\text { iluminación. }\end{array}$} & $\begin{array}{l}\text { (Molina } \\
\text { Grima et al., } \\
\text { 1999) }\end{array}$ \\
\hline $\begin{array}{l}\text { Sistema } \\
\text { airlift }\end{array}$ & A-M & $\mathrm{M}$ & A & B & $\mathrm{R}$ & & & $\begin{array}{c}\text { (Reyna- } \\
\text { Velarde } \text { et al., } \\
\text { 2010) }\end{array}$ \\
\hline $\begin{array}{l}\text { Columna } \\
\text { de burbu- } \\
\text { jeo }\end{array}$ & $\mathrm{M}$ & $\mathrm{M}$ & $\mathrm{M}$ & B & $\mathrm{R}$ & & & $\begin{array}{c}\text { (Tredici y } \\
\text { Zittelli, 1998) }\end{array}$ \\
\hline $\begin{array}{l}\text { Superficie } \\
\text { plana }\end{array}$ & A & A & A & B-M & $\mathrm{D}$ & $\begin{array}{l}\text { Alta productivi- } \\
\text { dad de biomasa, } \\
\text { económico, fácil } \\
\text { de limpiar, baja } \\
\text { acumulación de } \\
\text { oxígeno. }\end{array}$ & $\begin{array}{l}\text { Dificultad en } \\
\text { el control de } \\
\text { temperatura, } \\
\text { cierto grado de } \\
\text { crecimiento de } \\
\text { la pared. }\end{array}$ & $\begin{array}{c}\text { (Tredici y } \\
\text { Zittelli, 1998) }\end{array}$ \\
\hline Tubular & A & A & B-A & B-A & $\mathrm{R}$ & $\begin{array}{l}\text { Adecuada para } \\
\text { cultivos al aire li- }\end{array}$ & $\begin{array}{l}\text { Formación de } \\
\text { gradientes de } \\
\text { pH, disolución }\end{array}$ & $\begin{array}{l}\text { (Henrard et } \\
\text { al., 2011) }\end{array}$ \\
\hline Hibrido & A-M & A & A-M & B & $\mathrm{R}$ & $\begin{array}{l}\text { bre, alta producti- } \\
\text { vidad, económico }\end{array}$ & $\begin{array}{l}\text { de oxígeno y } \\
\mathrm{CO}_{2} \text { a lo largo } \\
\text { del tubo. }\end{array}$ & $\begin{array}{c}\text { (Lee et al., } \\
\text { 1995) }\end{array}$ \\
\hline
\end{tabular}

A: alto; M: medio; B: bajo; D: difícil; R: razonable

para el tratamiento de digestión anaeróbica de desechos porcinos; el fotobiorreactor cerrado mostró mejor desarrollo en términos de captación de nitrógeno para producción de biomasa y utilización de materia orgánica para crecimiento celular vía absorción de $\mathrm{CO}_{2}$.

Henrard et al. (2011), estudiaron la cinética de crecimiento de Cyanobium sp., en fotobiorreactor en modo semicontinuo, ya que muchas veces los cultivos de cianobacterias a gran escala no son económicamente viables para ser desarrollados por alimentación discontinua, obtuvieron alta productividad en la velocidad especifica de crecimiento, y lograron mantener al microorganismo en crecimiento exponencial durante 55 días.

Sistemas económicos como bolsas de polietileno se han utilizado bajo esta configuración, proporcionando alta transparencia, buena esterilización y con capacidad de aumentar la concentración celular tres veces más que los estanques abiertos (Carvalho et al., 2006). Su escalamiento no es sencillo, para proveer suficiente volumen de cultivo y tener eficiente velocidad de transferencia de gas, el diámetro del tubo debería ser alto al compararlo con sistemas como el de superficie plana (flat plate) o el tubular con loop, este requerimiento disminuye la relación $\mathrm{S} / \mathrm{V}$ restringiendo la eficiencia fotosintética.
B. Tubulares horizontales. Este tipo puede presentar varios diseños (Figura 1C). Su forma ofrece buenas ventajas en relación a los sistemas abiertos debido a su orientación hacia el sol lo que resulta en una alta y eficiente conversión de luz. En este tipo de reactores la transferencia de gas se lleva a cabo en las conexiones de los tubos o en una unidad especial dedicada al intercambio de gas. Estos sistemas pueden manejar volúmenes grandes ya que son menos susceptibles a la contaminación. Una desventaja de este tipo de reactores es que pueden generar cantidades considerables de calor.

Los métodos adaptados para el enfriamiento de estos sistemas han sido rociar con agua, solapamiento de tubos, sumergir en piscinas con agua a temperaturas controladas y regular la temperatura de la alimentación de recirculación (Molina et al., 1999).

Tredici y Zittelli (1998) diseñaron un fotobiorreactor horizontal con inclinación de pocos grados hacia el sol que ayuda a mejorar la eficiencia; consistía en un tubo de plexiglass de $3.4 \mathrm{~cm}$ de diámetro interno colocado uno al lado del otro sin espacio entre tubos. Los tubos conectados en la parte superior e inferior, con disposición de $5-7^{\circ}$ relativo al plano horizontal. La productividad volumetría y eficiencia fotosintética fue mayor que el sistema de superficie plana. 
El calor se controló rociando agua en el reactor cuando la temperatura del cultivo excedía un valor preestablecido.

Arbib et al. (2013) compararon a escala piloto, el raceway abierto $y$ el fotobiorreactor tubular airlift, localizados en una planta convencional de aguas servidas, con el objeto de realizar tratamiento terciario de efluente y mejorar la generación de algas. El fotobiorreactor tubular presentó mejor actividad fotosintética y productividad $21.76 \pm 0.3 \mathrm{~g} \mathrm{SS} \mathrm{m}^{-2} \mathrm{~d}^{-1}$, remoción total de nitrógeno $65.12 \pm 2.87 \%$, fósforo total $58.78 \pm 1.17 \%$ y lípidos de $20.80 \pm 0.22 \%$.

C. Tubulares helicoidales. El más frecuentemente usado es el Biocoil, compuesto por un set de tubos de polietileno ( $3 \mathrm{~cm}$ de diámetro interno) enrollados en un armazón circular abierto, acoplado a una torre de intercambio de gas y a un intercambiador de calor. Una bomba centrífuga hace llegar el medio de cultivo hasta la torre de intercambio de gas (Figura 1D) (Carvalho et al., 2006). Travieso et al. (2001) utilizaron y mejoraron el diseño original, siendo actualmente uno de los más eficaces entre otras cosas por tener una alta relación $\mathrm{S} / \mathrm{V}$ con poca extensión de terreno.

Ellos realizaron un escalamiento sencillo que consistió en agregar más unidades paralelas de capas de tubo manteniendo la carga hidráulica sin cambios. Posee una entrada de energía baja y una alta velocidad de mezcla mediante un procedimiento de entrada de gas pulsada.La colocación de una fuente de luz mediante el uso de un tubo interno, proporciona un control razonable de la intensidad de luz y una trayectoria de luz corta que mantiene la temperatura correcta. Lograron resultados satisfactorios en el crecimiento de Spirulina platensis, con productividades de $0.40 \mathrm{~g} \mathrm{~L}^{-1} \mathrm{~d}^{-1}$ sin daños celulares.

Soletto et al. (2008) evaluaron un fotobiorreactor helicoidal a escala de laboratorio con alimentación semicontinua en cultivo de Spirulina platensis bajo diferentes condiciones de intensidad de luz y velocidad de alimentación de $\mathrm{CO}_{2}$, para evitar la adición en exceso del $\mathrm{CO}_{2}$ al comienzo del cultivo que podría causar una inhibición por saturación de sustrato. La óptima velocidad de alimentación de $\mathrm{CO}_{2}$ para el crecimiento celular, se correlacionó con la intensidad de luz a la cual fue expuesta.

En general, el comportamiento de $S$. platensis estuvo más influenciado por patrones de alimentación de $\mathrm{CO}_{2}$ en baja actividad fotosintética. La velocidad del flujo de recirculación fue $1.0 \mathrm{~L} \mathrm{~min}^{-1}$, el número de Reynolds dentro del fotobiorreactor entre 400-1900, resaltando la ocurrencia de régimen laminar capaz de asegurar satisfactoriamente el crecimiento celular y prevenir estrés mecánico y oxidativo.

\section{Fotobiorreactores de superficie plana (flat plate)}

Están diseñados para hacer un uso eficiente de luz solar, sus paneles están construidos de tal manera que la relación $\mathrm{S} / \mathrm{V}$ es grande. Este tipo de fotobiorreactores pueden ser organizados de tal forma que estén orientados hacia el sol, lo cual permite mejor eficiencia en términos de energía absorbida (Carvalho et al., 2006) (Figura 1E). Puede ser de material transparente como vidrio, plexiglass, policarbonato. La agitación puede ser por burbujeo de aire por uno de sus lados a través de un tubo perforado o por rotación mecánica a través de un motor. Fue construido por Barbosa et al. (2005), utilizando policarbonato y acero inoxidable, teniendo una relación $\mathrm{S} / \mathrm{V}$ de $0.34 \mathrm{~cm}^{-1}$. Para la iluminación contaba con 10 tubos fluorescentes.

El fotobiorreactor puede escalarse organizando varias placas de superficie para ganar área, pero el alargamiento de la placa no es recomendado para el escalamiento, es mejor aumentar la altura del líquido y la ampliación de la trayectoria de la luz (Zhang et al., 2002). Degen et al. (2001) mostraron un fotobiorreactor flat plate con sistema airlift y tabiques adjuntados alternativamente en la parte delantera y la parte posterior de las caras mayores de la placa. Una chaqueta transparente de enfriamiento fue colocada en el lado iluminado delante del reactor. La productividad volumétrica de biomasa fue 1.7 veces más alta que un modelo similar pero con sistema de burbujeo.

\section{Fotobiorreactores tipo fermentador de tanque agitado}

Este tipo de reactor es el más convencional, y la agitación se da mecánicamente por un impulsor de diferentes tamaños y formas. Los deflectores reducen el efecto torbellino. Al fondo del fotobiorreactor se introduce la fuente de carbono, aire enriquecido con $\mathrm{CO}_{2}$, para el crecimiento de cianobacterias (Figura 1F). Se debe adaptar un sistema de iluminación interna que permita la distribución homogénea de luz, una desventaja del sistema es la baja relación $\mathrm{S} / \mathrm{V}$ que provoca disminución de eficiencia fotosintética. Es posible tener cultivos axénicos por lo que es indicado para obtener metabolitos de alto valor agregado (Carvalho et al., 2006).

\section{Fotobiorreactores tipo híbridos}

Muy usados ya que fusionan dos tipos de reactores haciendo que se complemente las ventajas y desventajas de cada uno respectivamente. Fernández et al. (2001), fusionaron un sistema airlift y uno tubular con loop externo, colocados de manera horizontal en una piscina con agua temperada, el fotobiorreactor tenía capacidad de 200 L. La disposición tubular de los loop ofrecía alta relación S/V para la recolección de luz y control de la 
temperatura del cultivo. El sistema airlift funcionaba como un desgasificador y controlador de velocidad del flujo a través del circuito. La ventaja es que incluye un mejor control sobre variables del cultivo, permitiendo mayor productividad y reducción de consumo energético (Fernández et al., 2001). Otro sistema híbrido de fotobiorreactor, fue desarrollado por Lee et al. (1995), donde el tanque de cultivo fue levantado 5 $\mathrm{m}$ del suelo, y tuberías de policloruro de vinilo (PVC) con inclinación de $25^{\circ}$ (en la horizontal) permitiendo al cultivo fluir hacia abajo para llegar a otro conjunto de tubos con dirección al tanque que está arriba, esto impulsado por un set de aire, para luego repetir el proceso hacia otro conjunto de tubos (Figura 1G).

\section{Algunas modificaciones en fotobiorreactores para cianobacterias}

Durante el proceso de producción de hidrógeno en algunas especies de cianobacterias heterocisticas, las nitrogenasas incorporadas en los heterocistos catalizan la reacción para formar, entre otras cosas, $\mathrm{H}_{2}$. Por $\mathrm{su}$ parte, la pared del heterocisto protege a la enzima del paso de oxígeno al cual es sensible. Existen algunos problemas hacia la rentable producción de hidrógeno, ya que el $\mathrm{H}_{2}$ producido por las cianobacterias en el reactor, es consumido rápidamente al interaccionar con el $\mathrm{N}_{2}$ (Markov, 2012). Por esto, se ha diseñado un fotobiorreactor capaz de reducir la concentración de nitrógeno molecular en el aire entrante, creando un vacío parcial de 250-300 torr logrando mayores tasas de producción de biomasa, por lo que un fotobiorreactor para este fin en cultivos de cianobacterias heterocisticas, debe ser capaz de crear vacío de manera controlada; ya que como se mencionó las nitrogenasas están en heterocistos y no se afectan por el oxígeno que en otras circunstancias solo con introducir una mezcla de aire y oxígeno se detendría el efecto enzimático (Markov, 2012). Además, para la producción de algunos productos específicos de cianobacterias como las ficobiliproteinas, Zeng et al. (2012) desarrollaron un nuevo fotobiorreactor con dimensiones y materiales detallados, en el cual las entradas de flujo de aire y $\mathrm{CO}_{2}$ podían ser programados de manera intermitente, esto logró aumentos significativos de ficocianina y biomasa.

Por otra parte algunos diseños de fotobiorreactores incorporan métodos de recuperación de biomasa $\mathrm{y}$ metabolitos como un paso continuo y progresivo del fotobiorreactor para tener un proceso más óptimo y rentable, debido a la condición procariota de las cianobacterias que le confiere un tamaño celular más pequeño, se debe incorporar la microfiltración para mayor eficiencia de recuperación en lugar de la centrifugación directa como sería en organismos eucarióticos (Molina et al., 2003). Además, muchas veces y dependiendo del metabolito que se quiera obtener, los cultivos se deben desarrollar en modo discontinuo, así el tiempo requerido para la carga, descarga y limpieza de fotobiorreactor es crucial, la condición procariota de cianobacterias y consiguiente menor tamaño celular, hacen que el diseño del fotobiorreactor deba evitar en su interior cualquier protuberancia, corrugues, conexión, entre otros, que propicie la persistencia del microorganismo, limitando el proceso en términos de tiempo de empleo para un nuevo lote.

\section{Conclusiones}

1 diseño y desarrollo de fotobiorreactores para
producción máxima de cianobacterias es muy complejo y requiere un conocimiento detallado de aspectos como transferencia de masa, estrés de cizallamiento, distribución de luz, escalamiento y biología del microorganismo. Además de la producción máxima, otros factores necesitan optimizarse como el diseño, relación costo beneficio, espacio físico, costos de mantenimiento y requerimientos energéticos. Hasta la fecha, ninguno de los fotobiorreactores cumple todos los requisitos. Existen métodos para superar las desventajas que afectan los fotobiorreactores existentes y desarrollar reactores innovadores que tomen en cuenta las necesidades de producción, los productos energéticos y las condiciones geográficas existentes.

\section{LiTERATURA CITADA}

Arbib, Z., J. Ruiz, P. Álvarez-Díaz, C. Garrido-Pérez, J. Barragan, J. Perales. 2013. Long term outdoor operation of a tubular airlift pilot photobioreactor and a high rate algal pond as tertiary treatment of urban wastewater. Ecol. Eng. 52: 143-153.

Barbosa, M. J., J. Hoogakker, R. Wijffels. 2003. Optimisation of cultivation parameters in photobioreactors for microalgae cultivation using the A-stat technique. Biomol. Eng. 20: 115-123.

Barbosa, M. J., W. Zijffers, A. Nisworo, W. Vaes, V. Schoonhoven, R. Wijffels. 2005. Optimization of biomass, vitamins, and carotenoid yield on light energy in a flat-panel reactor using the A-stat technique. Biotechnol. Bioeng. 89(2): 233-242.

Becker, W. 2004. Microalgae in human and animal nutrition. In: Richmond A, editor. Handbook of microalgal culture. Oxford: Blackwell Publishing; p. 312-51.

Bhagavathy, S., P. Sumathi. 2012. Evaluation of antigenotoxic effects of carotenoids from green algae Chlorococcum humicola using human lymphocytes. Asian Pacific Journal of Tropical Biomedicine 2(2): 109-117. 
Cano E., V. Blas-Valdivia, R. Rodríguez, P. Torres, M. Franco, A. Hernández, R. Ortiz. 2012. Uso terapéutico de algunos microorganismos, microalgas, algas y hongos. Rev Mex Cienc Farm. 43(4): 22-30.

Carvalho, A. P., L. Meireles, F. Malcata. 2006. Microalgal Reactors: A Review of Enclosed System Designs and Performances. Biotechnol Progr. 22: 1490-1506.

Castine, S., N. Paul, M. Magnusson, M. Bird, R. de Nys. 2013. Algal bioproducts derived from suspended solids in intensive land-based aquaculture. Bioresour Technol 131: 113-120.

Chen, C. Y., K. Yeh, R. Aisyah, D. Lee, J. Chang. 2011. Cultivation, photobioreactor design and harvesting of microalgae for biodiesel production: A critical review. Bioresour. Technol. 102: 71-81.

Chini, G., F. Lavista, A. Bastianini, L. Rodolfi, M. Vincenzini, M. Tredici. 1999. Production of eicosapentaenoic acid by Nannochloropsis sp. cultures in outdoor tubular photobioreactors. J. Biotechnol. 70: 299-312.

Chisti, Y. 2007. Biodiesel from microalgae. Biotechnol. Adv. 25: 294-306.

Chisti, Y., U. Jauregui-Haza. 2002. Oxygen transfer and mixing in mechanically agitated airlift bioreactors. Biochem. Eng. J. 10: 143-153.

Conroy, K., I. Davidson, M. Warnock. 2011. Pathogenic obesity and nutraceuticals. Proc Nutr Soc. 70(4): 426-438.

Datla, P. 2011. The wonder molecule called phycocyanin. Chennai-India: Parry Nutraceuticals (online). Retrieved February 18, 2012. Available http:/www.valensa.com/images3/Phycocyanin The $\% 20$ Wonder $\% 20$ Molecule.pdf

de Morais, M. G., J. Costa. 2007. Biofixation of carbon dioxide by Spirulina sp. and Scenedesmus obliquus cultivated in a three-stage serial tubular photobioreactor. J. Biotechnol. 129: 439-45.

Degen, J., A. Uebele, A. Retze, U. Schmid-Staiger, W. Trosch. 2001. A novel airlift photobioreactor with baffles for improved light utilization through the flashing light effect. J. Biotechnol. 92: 89-94.

Fernández, G., J. Sevilla, J. Pérez, E. Grima, Y. Chisti. 2001. Airliftdriven external loop tubular photobioreactors for outdoor production of microalgae: assessment of design and performance. Chem. Eng. Sci. 56: 2721-2732.

Gallardo-Casas, C. A., E. Cano-Europa, G. LópezGalindo, V. Blas-Valdivia, R. Olvera-Ramírez, M. Franco-Colín, R. Ortiz-Butrón. 2011. Las ficobiliproteínas de Spirulina maxima y Pseudanabaena tenuis protegen contra el daño hepático y el estrés oxidativo ocasionado por el
Hg2+. Rev. Mex. Cienc. Farm. 41(2): 30-35.

González-Fernández, C., B. Molinuevo-Salces, M. C. García-González. 2010. Open and enclosed photobioreactors comparison in terms of organic matter utilization, biomass chemical profile and photosynthetic efficiency. Ecol. Eng. 36: 14971501.

Gupta, V., S. Ratha, A. Sood, V. Chaudhary, R. Prasanna. 2013. New insights into the biodiversity and applications of cyanobacteria (Blue-green algae)-Prospects and challenges. Algal Research 2: 79-97.

Henrard, A.A., M. de Morais, J. Costa. 2011. Vertical tubular photobioreactor for semicontinuous culture of Cyanobium sp. Bioresour. Technol. 102: 4897-4900.

Jacob-Lopes, E., C. Ferreira, T. Teixeira. 2008. Biomass production and carbon dioxide fixation by Aphanothece microscopica Nägeli in a bubble column photobioreactor. Biochem. Eng. J. 40: 2734.

Jacob-Lopes, E., C. Scoparo, L. Lacerda, T. Franco. 2009. Effect of light cycles (Night/day) on $\mathrm{CO}_{2}$ fixation and biomass production by microalgae in photobioreactors. Chem. Eng. Process. 48: 306310.

Jaiswal, P., A. Kashyap. 2002. Isolation and characterization of mutants of two diazotrophic cyanobacteria tolerant to high concentrations of inorganic carbon. Microbiol. Res. 157: 83-91.

Janssen, M., M. de Winter, J. Tramper, L. Mur, J. Snel, R. Wijffels. 2000. Efficiency of light utilization of Chlamydomonas reinhardtii under mediumduration light/dark cycles. J Biotechnol 78: 12337.

Kumar, K., C. Dasgupta, B. Nayak, P. Lindblad, D. Das. 2011. Development of suitable photobioreactors for $\mathrm{CO}_{2}$ sequestration addressing global warming using green algae and cyanobacteria. Bioresour. Technol. 102: 4945-4953.

Kwon, J. H., G. Bernát, H. Wagner, M. Rögner, S. Rexroth. 2013. Reduced light-harvesting antenna: Consequences on cyanobacterial metabolism and photosynthetic productivity. Algal Research 2: 188-195.

Lee, Y. K., S. Ding, C. Low, Y. Chang, W. Forday, P. Chew. 1995. Design and performance of an $\alpha$-type tubular photobioreactor for mass cultivation of microalgae. J. Appl. Phycol. 7:47-51.

Loke, M. F., S. Lui, B. Ng, M. Gong, B. Ho. 2007. Antiadhesive property ofmicroalgal polysaccharide extract on the binding of Helicobacter pylori to gastric mucin. FEMS Immunol. Med. Microbiol. (50): 231-23. 
Markou, G., D. Georgakakis. 2011. Cultivation of fi lamentous cyanobacteria (Blue-green algae) in agro-industrial wastes and wastewaters: A review. Applied Energy 88: 3389-3401.

Markov, S. 2012. Hydrogen production in bioreactors: current trends. Energy Procedia 29: 394-400

Melnicki, M. R., G. Pinchuk, E. Hill, L. Kucek, S. Stolyar, J. Fredrickson, A. Konopka, A. Beliaev. 2013. Feedback-controlled LED photobioreactor for photophysiological studies of cyanobacteria. Bioresour. Technol. 134: 127-133.

Molina, E., E. Belarbi, F. Acién, A. Robles, Y. Chisti. 2003. Recovery of microalgal biomass and metabolites: Process options and economics. Biotechnol. Adv. 20: 491-515

Molina, E., F. Acién, F. García, Y. Chisti. 1999. Photobioreactors: light regime, mass transfer, and scaleup. J. Biotechnol. 70: 231-247.

Pérez-García O., F. Escalante, L. de-Bashan, Y. Bashan. 2011. Heterotrophic cultures of microalgae: metabolism and potential products. Water Res. 45(1): 11-36.

Posten, C., G. Schaub. 2009. Microalgae and terrestrial biomass as source for fuels- a process view. J. Biotechnol. 142(1): 64-69.

Pruvost. J., L. Pottier, J. Legrand. 2006. Numerical investigation of hydrodynamic and mixing conditions in a torus photobioreactor. Chem. Eng. Sci. 61: 4476-4489.

Raposo, M., R. Morais, A. Morais. 2013. Health applications of bioactive compounds from marine microalgae. Life Sciences. (93): 479-486

Reinhold, L., M. Sviman, A. Kaplan. 1991. A model for inorganic carbon fluxes and photosynthesis in cyanobacterial carboxisomes. Can. J. Bot. 69: 984-988.

Reyna-Velarde, R., E. Cristiani-Urbina, D. Hernández-Melchor, F. Thalasso, R. CañizaresVillanueva. 2010. Hydrodynamic and mass transfer characterization of a flat-panel airlift photobioreactor with high light path. Chem. Eng. Process: Process Intensification 49(1): 97-103.

Sánchez, A., F. García, A. Contreras, E. Molina, Y. Chisti. 2000. Bubble-Column and Airlift Photobioreactors for Algal Culture. AIChE J. 46(9): 1872-1887.

Sato, T., D. Yamada, S. Hirabayashi. 2010. Development of virtual photobioreactor for microalgae culture considering turbulent flow and flashing light effect. Energy Convers.Manage 51(6): 1196-1201.

Sepúlveda, A. 2011. Crecimiento de cianobacterias en fotobiorreactores con iluminación controlada. Tesis para obtener el grado de Maestro en Ciencias en Bioprocesos. Instituto Politécnico Nacional,
México.

Sharma, M. K., A. Sharma, A. Kumar, M. Kumar. 2007. Evaluation of protective efficacy of Spirulina fusiformis against mercury induced nephrotoxicity in Swiss albino mice. Food and Chem Toxicol. 45(6): 879-887.

Singh, R. N., S. Sharma. 2012. Development of suitable photobioreactor for algae production - A review. Renewable and Sustainable Energy Reviews 16(4): 2347-2353.

Soletto, D., L. Binaghi, L. Ferrari, A. Lodi, J. Carvalho, M. Zilli, A. Converti. 2008. Effects of carbon dioxide feeding rate and light intensity on the fed-batch pulse-feeding cultivation of Spirulina platensis in helical photobioreactor. Biochem. Eng. J. 39: 369-375.

Tamburic, B., F. Zemichael, P. Crudge, G. Maitland, K. Hellgardt. 2011. Design of a novel flat-plate photobioreactor system for green algal hydrogen production. Int. J. Hydrogen Energy 36: 65786591.

Torzillo, G., B. Pushparaj, J. Masojidek, A. Vonshak. 2003. Biological constraints in algal biotechnology. Biotechnology and bioprocess engineering 8: 338348.

Travieso, L., D. Hall, K. Rao, F. Benítez, E. Sánchez, R. Borja. 2001. A helical tubular photobioreactor producing Spirulina in a semicontinuous mode. Int. Biodeterior. Biodegrad. 47: 151-155.

Tredici, M. R., G. Zittelli. 1998. Efficiency of sunlight utilization: tubular versus flat photobioreactors. Biotechnol. Bioeng. 57(2): 187-197.

Ugwu, C. U., J. Ogbonna, H. Tanaka. 2002. Improvement of mass transfer characteristics and productivities of the inclined tubular photobioreactors by installation of internal static mixers. Appl. Microbiol. Biotechnol. 58:600-607.

Zeng, X., M. Danquah, S. Zhang, X. Zhang, M. Wu, X. Chen, I-S. Ng, K. Jing, Y. Lu. 2012. Autotrophic cultivation of Spirulina platensis for $\mathrm{CO}_{2}$ fixation and phycocyanin production. Chem. Eng. J. 183: 192-197.

Zhang, K., N. Kurano, S. Miyachi. 2002. Optimized aeration by carbon dioxide gas for microalgal production and mass transfer characterization in a vertical flatplate photobioreactor. Bioproc Biosyst Eng 25: 97-101.

Zijffers, J. W., M. Janssen, J. Tramper, R. Wijffels. 2008. Design process of an area-efficient photobioreactor. Mar. Biotechnol. 10: 404-415. 